\title{
The role of attention in flavour perception
}

\author{
Richard J Stevenson
}

\begin{abstract}
Flavour results primarily from the combination of three discrete senses: taste, somatosensation and olfaction. In contrast to this scientific description, most people seem unaware that olfaction is involved in flavour perception. They also appear poorer at detecting the olfactory components of a flavour relative to the taste and somatosensory parts. These and other findings suggest that flavour may in part be treated as a unitary experience. In this article, I examine the mechanisms that may contribute to this unification, in particular the role of attention. Drawing on recent work, the evidence suggests that concurrent gustatory and somatosensory stimulation capture attention at the expense of the olfactory channel. Not only does this make it hard to voluntarily attend to the olfactory channel, but it also can explain why olfaction goes largely unnoticed in our day-to-day experience of flavour. It also provides a useful framework for conceptualizing how the unitary experience of flavour may arise from three anatomically discrete sensory systems.
\end{abstract}

\section{Introduction}

'Flavour' refers to the perceptual experience we have when we eat and drink [1-3]. In the mouth, three anatomically discrete sensory systems contribute to flavour: taste, olfaction and somatosensation [4]. Taste, which is detected by receptors primarily located on the surface of the tongue, generates sensations of sweetness, sourness, bitterness, saltiness, meatiness (umami) and possibly other sensations, too, relating to fattiness and metallic tastes [5]. Olfaction detects the volatile chemicals that are released by food and drink in the mouth, especially during chewing. These volatiles may be pumped via the nasopharynx to the olfactory receptors located in the nasal vault and/or may be carried by exhaled air routed via the nose when the mouth is full [6,7]. In contrast to taste, olfaction has a large range of sensations associated with it and is a major contributor to our experience of flavour [8]. The final contributory sense is somatosensation. Not only is this instrumental in generating our sense of food texture via receptors located within the various tissues of the mouth [9], but it also detects sensations relating to temperature, irritation and pain. While the range of sensation that the somatosensory system provides is almost certainly greater than taste, it is probably less than for olfaction.

Correspondence: dick.stevenson@mq.edu.au

Department of Psychology, Macquarie University, Sydney, NSW, 2109, Australia
While somatosensation, olfaction and taste comprise the senses involved when a food is in the mouth, the experience of flavour can also be affected by other properties of the food that are perceived during or just prior to ingestion. During ingestion, the sound that a food makes when it is being chewed can influence our experience of texture, as may sound prior to ingestion, although its influence is probably fairly minor $[10,11]$. Far more potent are the effects of the appearance of the food and the expectations that these visual cues can generate [12]. Numerous studies have indicated that appearance can affect both the enjoyment of that food as well as the way in which it is perceived when subsequently ingested [13,14].

This modality-based description of the senses involved in flavour perception does not seem to be in accord with most people's day-to-day experience of flavour, or with contemporary functionalist theories of flavour perception $[1,3]$, which emphasise the need to collate information about a single act (eating) into a single percept: a unitary flavour experience (or perhaps a gestalt). Flavour, then, seems to be something of an emergent property from the individual senses (taste, olfaction and somatosensation) that make it up. The aim of this article is to critically examine this 'unitariness' claim and to explore its psychological basis, especially with respect to the role of attention.

\section{Is flavour a 'unitary' experience?}

A magenta-coloured circle, while similar to its primary component colours of red and green, has nonetheless a 
unitary character. The combination of red and green are not directly evident, and something new emerges: magenta. Flavour does not appear to meet this 'strong' form of unitariness, as it is quite evident that people have some capacity to accurately decompose flavour into its component elements [15-17]. However, this process of decomposition seems to be more readily achievable for some of the senses that compose flavour than for others. For taste, combinations of tastants within a mixture can generally be identified individually and similarly when tastes are mixed with odourants and presented to the mouth [17]. For somatosensory stimuli, participants can generally detect most of the characteristic textures of a food, and these do, by and large, correlate with physically derived measures of texture properties [18]. Although not a lot is known about the ability to detect individual texture components when tastes and smells are present, it would seem that texture perception could occur independently and successfully. The notable exception would seem to be olfaction. In simple mixtures of tastes and smells (which also have a somatosensory component generated by the mixtures' physical presence in the mouth), all of the components can usually be identified; however, with increasing mixture complexity (that is, more odourants and more tastes), this capacity to identify individual odours diminishes far more rapidly than for taste $[16,17]$. That is, odours appear to be both harder to discriminate from other odours (as just the number of odours increase) and from tastes (as just the number of tastes increase).

These findings raise two issues relevant to the question of the unitariness of flavour perception. First, in all of the studies described above, participants were deliberately asked to adopt an 'analytical stance'; that is, participants were required to attempt to deconstruct the flavour stimulus. Importantly, this analytical stance may not be the way in which people normally perceive flavour during ingestion; indeed all these findings tell us is that people can fairly readily decompose the taste and somatosensory components, and to some extent the olfactory ones as well, when they are instructed to do so. The second issue is that odours seem to be much harder to identify and detect in a mixture with tastes (and possibly in mixtures with multiple somatosensory components, too, but here the evidence is just suggestive; see Bult et al. [19]). This may suggest some special difficulty in identifying individual odours when they arise from the mouth.

Several other lines of evidence also converge on the conclusion that people do not routinely notice odours as being component parts of flavour. In a series of studies, Rozin [20] examined the words that people use to describe flavour experiences. First, he examined whether any major language had a special word that identified a role for olfaction during eating and drinking. In all of the seven major languages examined, none had a term that explicitly acknowledged the olfactory component of flavour. However, like English, many used terms such as 'taste' and 'flavour' to describe the experience of food in the mouth. Rozin [20] also had people judge a series of statements to see whether in each case the term 'taste' or 'flavour' was best suited to describing the experience referred to in the statement. 'Taste' was widely used, even for items that had a major olfactory component. Although 'flavour' tended to be used more frequently for foods that had a much greater olfactory component, it was quite evident that most participants did not readily acknowledge the role of olfaction in flavour perception, and this is true of speakers of most other languages as well.

A further reason to suspect that participants do not routinely appreciate the role of olfaction in flavour perception comes from experiments in which its role is made evident to naïve participants. A simple demonstration of olfaction's role in flavour perception can be made by pinching the nose during eating and drinking. Pointing out the role of olfaction in this way seems to come as a surprise to most people, as several investigators have noted [21]. In a similar vein, when people visit a doctor with olfactory-related problems and describe their symptoms, it is common for them to state that they have both olfactory and taste problems [22-24]. However, upon investigation in nearly all cases, the problem is specific to olfaction and the taste system is largely intact, again indicating the absence of awareness of the role of olfaction in flavour perception [23]. The same conclusion may be drawn from other quite different findings. In our recent work on olfactory synaesthesia, where colours are induced by particular odours, we noted the almost total absence of synesthetic experiences induced by flavour (RJ Stevenson, A Russell, A Rich, unpublished data). This also seems to be the case for individuals who experience olfactory hallucinations, in that reports of hallucinatory flavours are either rare or are described as being gustatory hallucinations instead [25].

The findings described above suggest that the contribution of olfaction to flavour goes largely unnoticed, and even when participants are asked to detect its presence, this may be more difficult than for taste and somatosensation. Together these data may suggest some form of unitization of olfaction with taste and somatosensation. A more contentious proposition is that the default position for experiencing flavour is as a whole, in contrast to when participants are asked to analyse their experience as a series of individual parts. While this idea of dual levels of experience is not unique (see Kubovy and Van Valkenburg [26], who describe music perception as an example of a preservative (the sound of 
individual instruments) emergent property (the tune)), whether the default position really is the 'flavour' overall remains to be demonstrated. Nonetheless, there clearly is a question to answer regarding the unitization of olfaction, and the following section examines how this might occur.

\section{Odour unitisation into flavour}

A rather obvious explanation for unawareness of the role of olfaction in flavour perception is simply that we have not had the opportunity to learn about it. During development, most children discover that closing their eyes, blocking their ears or pinching their noses eliminates sight, hearing or smell accordingly. However, most children do not learn that we need a nose for 'tasting' food and drink. While we may lack general knowledge about the nose, and thus not appreciate its role in flavour perception, this would seem an incomplete explanation of olfactory unitisation for two reasons. First, knowing that the nose is involved in flavour perception may make no difference in the way we perceive flavour under routine conditions. The literature pertaining to this issue is somewhat problematic for the reason alluded to above, namely, that studies of experts (that is, those who know the role of the nose) have generally asked them to adopt an analytical stance. Although at best they may be a little better than naive participants [27], they do not seem to report any systematic differences in routine flavour perception. Indeed, if knowing about the nose did produce significant changes in flavour perception, presumably this would alter experts' capacity to generate successful wines, cheeses and other foods, and this does not seem to be the case. A second reason for thinking that not knowing the role of the nose in flavour may be the cause of our general lack of awareness of its role in flavour comes from the studies identified earlier, which show that even under analytic conditions, the capacity to detect odour components is limited relative to the other senses involved in flavour [15-17]. So, while lack of knowledge might at best assist unitization, knowing does not seem to make a great deal of difference.

A further contributor to olfactory unitisation may be nasal airflow direction. During orthonasal perception, or sniffing, odours ascend via the anterior nares to the olfactory receptors. In contrast, retronasal perception always involves the passage of air in the opposite direction, from the interior to the environment. There are at least two ways in which this difference in airflow direction can be detected by the body. The anterior and posterior areas of the nose differ in their sensitivity to somatosensory stimuli. The anterior portion is particularly sensitive to irritant stimuli [28]. Most odourants are to some extent irritants; that is, they are detected by the free nerve-ending receptors of the trigeminal nerve
[29]. In contrast, the posterior part of the nose is more richly innervated with mechanoreceptors, which may be sensitive to expulsions of air from the interior of the body (mouth or lungs) that occur when the velopharyngeal flap opens, allowing air into the posterior portion of the nose [28]. Not only may the nasal cavity itself be sensitive to airflow direction but so also may the olfactory epithelium. In this case, the same chemical may induce a different pattern of activation across the epithelium, depending upon the direction in which it travels over its surface [30]. In sum, the direction of airflow provides an important cue regarding the likely source of the odour.

Several studies have indicated that airflow cues can shift the apparent locus of an odour [31]. Using endoscopically placed catheters, one just inside the nose and another near the back of the throat, revealed that odourants released via the former route are attributed to the nose (that is, coming from the external environment), whereas those from the latter route are experienced as arising in the mouth [32]. But what does it mean to make such a judgment that an odour is perceived to be located in one location or another? This question is very hard to answer, because, if taken at face value, it would suggest that participants are as readily able to detect an odour in the mouth (that is, judging that this is where it comes from) as they are an odour in the nose. Puzzlingly, this would seem to contradict the findings reviewed above, namely, that naïve participants do not know that odour is involved in flavour, and, ergo, they should not be aware that odours may arise in their mouths to stimulate their olfactory receptors. One way out of this apparent paradox is to assume that the experience of having an odour delivered to the back of the throat is surrounded by considerable uncertainty about what is being experienced, but that location of the experience is still evident (that is, I know where it is, but not what it is). In contrast, odours delivered to the anterior nares may be understood to be odours partly because of their perceived location. In terms of the role of nasal airflow in generating unitary flavour percepts and in allowing smell to go unrecognized by most people as part of flavour, its importance may lie in identifying externally arising smells by directing attention to the 'tip of the nose' and the external environment, the location routinely associated with this sense modality.

If attention to the nose is important in knowing that an experience is olfactory and arises from the external world, then this raises the possibility that not attending to the olfactory channel during retronasal perception may be instrumental in not knowing that odours are involved in flavour perception. Indeed, it could be that our capacity to attend to the olfactory channel when the source of odour is in the mouth, rather than at the tip 
of the nose, is constrained in some way. For example, it has been argued that sniffing acts in a somewhat similar way to shifting one's gaze to a point in space that one wishes to see (see Mainland and Sobel [33]). That we cannot readily 'sniff' a retronasal odour (or at least that we do not normally do this, but we may do it in some way that is yet to be documented) might suggest one reason why we are not as readily able to attend to an odour in the mouth as we can to taste or a somatosensory stimulus. A further possibility, and one that has been missing in the discussion so far, is the role of simultaneously present oral stimulation from taste and somatosensation, which has been recognized before as a factor likely to be important in flavour unitisation [3]. These senses may be more effective than olfaction at capturing attention, and somatosensation may itself be important in causing tastes to be perceived as a property of the food rather than as a property of the tongue, although the role of attention in this process is not currently known $[34,35]$. If taste and somatosensation do result in attentional capture (at the expense of olfaction), this could make it difficult to shift attention away from these simultaneously present senses (for example, the burning of chilli pepper or the sweetness and smooth creaminess of mousse) to the olfactory channel. Before turning to discuss the implications of this, it is important to note the distinction between attending to a sense modality and attending to a spatial location. While these are both independently possible, here they may be especially entwined, the nose with olfaction and the mouth with taste and/or somatosensation.

Some recent experimental studies have started to shed light on the possible role of attention in olfactory localisation to the mouth and thus on the role that attention may have in accounting for (1) our lack of awareness of the role of odour in flavour, (2) our limited ability to attend to the olfactory channel when an odour forms part of an orally presented mixture and thus (3) the apparent unitisation of olfaction in flavour perception. In these studies, another technique was adopted to explore participants' experiences of 'where' an odour was perceived to be located [36-39]. In this case, the odour was always presented to the nose while a taste or other oral stimulus (for example, a tasteless viscous solution) was present in the mouth. Participants were then asked either to judge the likely source of the smell (that is, the jar they were sniffing or the fluid they had poured into their mouths) or to mark on a diagram the location where they felt the smell sensation was coming from. Regardless of the measurement method used, the results are remarkably consistent. When a taste is present in the mouth (and to a lesser extent other types of oral stimulation), participants are more likely to experience the odour as localized to the mouth; when the mouth is empty or when water is held still in the mouth, however, participants are more likely to experience the odour as arising from the nose and the external environment.

If we pose a question similar to the one we asked of the earlier set of studies using catheters to deliver odours to the nose (namely, What does it mean to say an odour is localized to the mouth?), we get a somewhat different answer. Under conditions where participants localise the odour to the mouth, they appear to judge the experience arising via their olfactory system as being part of, or one and the same with, the experience arising in their mouth from the taste or somatosensory stimulus held there. Another and perhaps more illuminating way of restating this is that participants under such conditions simply do not attend to the odour, but attend only to the oral stimulus. Consequently, when asked to judge where the 'odour' is, their attention, which is captured by events in the mouth, leads them to conclude that the mouth is the locus of stimulation.

If this attentional account is correct, then one would predict that if attention could be drawn to the nose, such judgments would change. Similarly, if events in the mouth were made even more attention-demanding, then participants would be even more prone to report odours as arising at that location. Both of these predictions have been confirmed [38]. If an irritant (glacial acetic acid) is added to an odour sniffed via the nose while a taste is held in the mouth, this olfactory combination is significantly less likely to be localized to the mouth than the odour alone (that is, without the irritant added). These findings are illustrated in Figure 1a. A similar effect (see Figure 1b) also occurs if the concentration of the odourant is increased [37]. Conversely, if a taste in the mouth is presented at a higher concentration, the likelihood that the concurrently sniffed odour will be localized to the mouth is increased (see Figure 1a). In all of these cases, the salience of the olfactory and oral events seem to compete to capture attention, and where the oral events are more salient, as is often the case, attention will be directed toward events in the mouth at the expense of events at the nose. That oral events are generally more salient reflects both the hedonic nature of gustatory stimulation (for example, sucrose is pleasant and bitter is unpleasant) and that the number of discretely perceivable events generated by taste and somatosensation may exceed those generated by olfaction. In addition, the rather obvious fact that we are placing food (or drink) into our mouths further serves to make the mouth the locus of attention during eating and drinking, at the expense of the nose.

Two additional sources of evidence have also emerged to support this type of attentional account. The first concerns individuals who have an impaired ability to selectively attend to the olfactory channel. Several studies have demonstrated that healthy participants can 

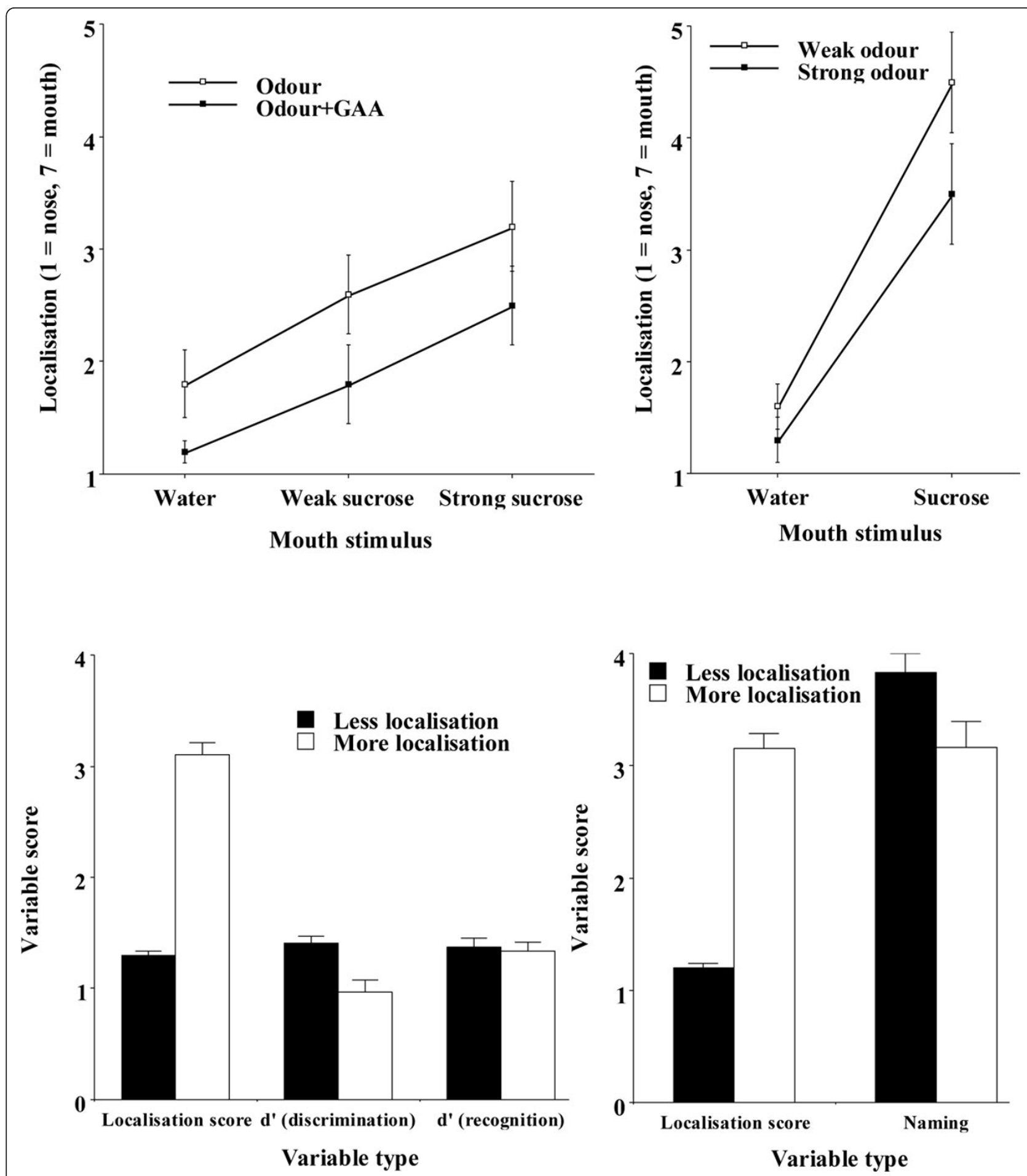

Figure 1 (a) Top left: Mean localisation rating (and standard error (SE)) for an odour alone and for the odour with the irritant glacial acetic acid (GAA) added when water as well as weak and strong concentrations of sucrose serve as the mouth stimuli. (b) Top right: Mean localisation rating (and SE) for a weak and strong odour (same odour with different concentrations) when water and sucrose serve as the mouth stimuli. (c) Bottom left: Mean localisation, discrimination d' and recognition d'scores (and SE), for participants demonstrating lesser or greater degrees of oral localisation. (d) Bottom right: Mean localisation and naming score (number correct/8 and SE) for participants demonstrating lesser or greater degrees of oral localisation. 
selectively attend to the olfactory channel on demand, at least when it involves sniffing odours in the external environment [40]. However, this type of ability appears to be impaired in people with damage to their mediodorsal nucleus of the thalamus (MDNT) [41]. While they are able to complete most olfactory tasks in a manner comparable to healthy participants, tasks that require that they selectively attend to the olfactory channel appear to be impaired $[42,43]$. If one recalls the design of the experiment described above, in which odour and taste concentrations were manipulated, increased odour concentrations tended to result in greater localisation toward the nose and increased taste concentration tended to result in greater localisation toward the mouth. These effects were independent, so if the capacity to attend to the nose is diminished, then one might expect that such a person would be far more sensitive to manipulations that favoured oral capture of attention. This prediction was confirmed in that localisation to the mouth, using the same type of procedure, was significantly greater in MDNT patients than in controls [43]. Moreover, the extent of this greater tendency to localise odours to the mouth was significantly associated with participants' capacity to attend to the olfactory channel on a test of selective attention. This would suggest that eliminating (or reducing) the capacity to attend to the olfactory channel results in greater oral localisation.

A final source of evidence arises from another prediction that can be derived from an attentional account of olfactory unitisation. If one cannot or does not attend to odours when they are localized to the mouth, then olfactory tasks that require attention should be more impaired when an odour is presented in this manner than olfactory tasks that do not require attention. To test this prediction, my research groupselected three olfactory tasks, two of which we believed would require attention and one of which would not. The tasks requiring attention were discrimination and odour naming, with odour learning (indexed by a later recognition memory test) included as the non-attention-demanding task. This last mentioned task was deemed non-attention-demanding, as a considerable body of evidence suggests that people can acquire various forms of olfactory knowledge (that is, which odours have been smelled, as well as odour-odour and odour-taste associations) without explicitly attending to the olfactory channel [44-46].

In this study [39], participants were asked to sniff an odour bottle while holding one of three fluids in their mouth: sucrose, water or a tasteless viscous solution. For the discrimination task, which also served as the exposure phase for the later surprise recognition memory test, participants were asked to sniff one odour with a solution in their mouth and then repeat this process for a second odour. Participants were asked not only to judge the perceived location of the odour (sniffing jar or fluid placed in the mouth) but also whether the first trial (sniff with solution in mouth) was the same as or different from the second trial (sniff with solution in mouth). As the solution in the mouth remained constant, the ability to detect a difference reflected the ability to discriminate one odour from another. A further task then followed, in which participants again sniffed an odour with a solution in their mouths, but this time they were asked not only to judge its location but also to select its name from a list provided. In the final phase of the experiment, participants were provided all of the odours used in the discrimination test, along with an equal number of new stimuli not previously encountered in the experiment. Their task was to sniff each one (with no solution present) and judge whether it was 'old' or 'new'.

As we expected, there was considerable individual variation in localisation, so, even when using sucrose, there were still participants who did not demonstrate localisation to the mouth. Even when using water there were participants who did experience oral localisation. For this reason, we treated localisation as a continuous variable and 'oral solution used' (that is, water vs. sucrose vs. viscous solution) as a dummy variable in a regression approach to determine whether perceived localisation was associated with performance on odour discrimination, naming and recognition memory (that is, odour learning). As expected, we observed significant associations between discrimination and naming performance, as well as degree of oral localization, and these are illustrated diagrammatically in Figures $1 \mathrm{c}$ and $1 \mathrm{~d}$. The more an odour was localized to the mouth, the poorer participants performed on tasks deemed likely to require attention, consistent with our expectation that localisation to the mouth involves capture of attention by concurrent oral events. However, recognition memory performance was unaffected by the degree of localisation (see Figure 1c) that a participant reported experiencing during the discrimination phase of the experiment (that is, where odours were exposed and learned).

These various findings suggest that unitary experience of olfaction in flavour is to some degree an attentional phenomenon. It is an attentional phenomenon in two ways. First, the failure of most people to know that olfaction is involved in flavour and the difficulty that they have in detecting the olfactory components of flavour may result from concurrent oral stimulation's capturing attention at the expense of olfaction. This would make it hard to notice that 'smell' was present, perhaps even more so if attentional capture by gustation and/or somatosensation is somehow especially 'sticky', making it hard to switch attention to the olfactory modality (see 
Spence et al. [47], who suggested that somatosensation is especially 'sticky'). This is likely to be further compounded by the difficulty in learning about the role of olfaction in flavour (that is, we do not learn this in childhood) and the fact that our olfactory attentional spotlight, sniffing, is (generally) unavailable to retronasal olfaction. More broadly, and this has not been well explored, we may not routinely attend to other dimensions of flavour, notably taste and somatosensation, during eating and drinking, although we can if we so choose, as the various analytical experiments noted earlier would suggest. Rather, we attend in a general manner to events in the mouth, but not in particular to events within a modality.

It is important to stress that while we now have some evidence directly supporting the first point, the second is still largely a matter of conjecture, so it is important to consider how both these attentional phenomena (especially the second) may be further explored. One interesting possibility in regard to the first point is that individuals with peripheral gustatory impairments (ageusia or hypogeusia) should demonstrate less oral capture than controls as a result of impaired concurrent oral stimulation. A similar prediction could also be made for individuals with impaired oral somatosensation. With respect to the second point (routine focus on flavour, not on the individual modalities), we might expect that attempts to explicitly recall details about a food or drink would be affected by the analytical stance taken during the earlier bout of eating and drinking. If attention is normally focused on the overall impression, flavour, then recollections of that experience should differ from those of participants asked to adopt a more analytical stance. While this type of approach has been used to study the effect of an analytical stance on odour-taste learning $[48,49]$, it has not, to my knowledge, been employed to study the general recollective experience of an eating episode.

\section{Conclusion}

In the Introduction, the suggestion was made that olfaction may become unitized into flavour, as implied by participants' failure to know the role of olfaction in flavour and by the finding that even when instructed to adopt an analytical stance and search for odour elements within a flavour mixture, they were poorer at this than they were at searching for somatosensory or gustatory parts. A more general suggestion was also made that during routine eating and drinking, attention may be directed at the whole experience rather than at particular parts of that experience. So far the discussion has largely avoided any attempt to deal with the specific type of attentional process that may be involved. Attention can be fractionated into endogenous (self-directed) and exogenous (stimulus-directed) forms [50,51]. As described above, Stevenson et al. [38] found that varying the stimulus characteristics so that either oral or nasal cues became more salient had the effect of shifting localisation toward the physical locus of the more salient cue. This would seem to suggest an effect of exogenous attention. In our more recent study [39], examining the impact of oral localisation on olfactory performance (that is, discrimination, naming and recognition memory), participants are likely to have tried attending to the olfactory channel. That performance here was poor for attention-demanding tasks when the odour was localised to the mouth would seem to suggest that under such conditions participants might not be readily able to voluntarily switch attention to the olfactory channel. Again, this would suggest the dominance of exogenous (that is, stimulus-driven) attentional processes. Therefore, one tentative conclusion is that exogenous attentional processes may be more important in generating oral localisation, and when engaged, these may be difficult to voluntarily override. This would seem to echo the findings of Ashkenazi and Marks [15], who reported that attempting to selectively attend to the olfactory component of a flavour did not significantly benefit performance (see also Veldhuizen et al. [52] for further confirmatory findings). More generally, this would suggest that it might be exogenous attentional processes that favour the unitization of olfaction into flavour.

\section{Acknowledgements}

The author thanks the Australian Research Council for their continued support.

\section{Authors' contributions}

RJS conceived and prepared the ideas presented here and drafted the manuscript.

\section{Competing interests}

The author declares that they have no competing interests.

Received: 4 May 2011 Accepted: 21 March 2012

Published: 21 March 2012

\section{References}

1. Auvray M, Spence C: The multisensory perception of flavor. Conscious Cogn 2008, 17:1016-1031.

2. Stevenson RJ: The Psychology of Flavour Oxford: Oxford University Press; 2009.

3. Small DM: Flavor and the formation of category-specific processing in olfaction. Chemosens Percept 2008, 1:136-146.

4. Simon SA, de Araujo IE, Gutierrez R, Nicolelis MAL: The neural mechanisms of gustation: a distributed processing code. Nat Rev Neurosci 2006, 7:890-901

5. Schiffman SS: Taste quality and neural coding: implications from psychophysics and neurophysiology. Physiol Behav 2002, 69:147-159.

6. Trelea IC, Atlan S, Déléris I, Saint-Eve A, Marin M, Souchon I: Mechanistic mathematical model for in vivo aroma release during eating of semiliquid foods. Chem Senses 2008, 33:181-192.

7. Hodgson M, Linforth RST, Taylor A: Simultaneous real-time measurements of mastication, swallowing, nasal airflow and aroma release. J Agric Food Chem 2003, 51:5052-5057. 
8. Dravnieks A: Atlas of Odor Character Profiles West Conshohocken, PA: American Society for Testing and Materials; 1985.

9. Christensen CM: Food texture perception. In Advances in Food Research Volume 29. Edited by: Chichester CO. Orlando, FL: Academic Press; 1984:159-199.

10. Vickers ZM, Bourne MC: A psychoacoustical theory of crispness. J Food Sci 1976, 41:1158-1164.

11. Zampini M, Spence C: The role of auditory cues in modulating the perceived crispness and staleness of potato chips. J Sens Stud 2004, 19:347-363.

12. Yeomans MR, Chambers $L$, Blumenthal $H$, Blake $A$ : The role of expectancy in sensory and hedonic evaluation: the case of smoked salmon icecream. Food Qual Prefer 2008, 19:565-573.

13. Shankar MU, Levitan CA, Spence C: Grape expectations: the role of cognitive influences in color-flavor interactions. Conscious Cogn 2010, 19:380-390.

14. Spence C, Levitan C, Shankar MU, Zampini M: Does food color influence taste and flavor perception in humans? Chemosens Percept 2010, 3:68-84

15. Ashkenazi A, Marks LE: Effect of endogenous attention on detection of weak gustatory and olfactory flavors. Percept Psychophys 2004, 66:596-608.

16. Laing DG, Link C, Jinks AL, Hutchinson I: The limited capacity of humans to identify the components of taste mixtures and taste-odour mixtures. Perception 2002, 31:617-635.

17. Marshall K, Laing DG, Jinks AL, Hutchinson I: The capacity of humans to identify components in complex odor-taste mixtures. Chem Senses 2006, 31:539-545.

18. Cardello AV, Maller O, Kapsalis JG: Perception of texture by trained and consumer panelists. J Food Sci 1982, 47:1186-1197.

19. Bult JHF, de Wijk RA, Hummel T: Investigations on multimodal sensory integration: texture, taste, and ortho- and retronasal olfactory stimuli in concert. Neurosci Lett 2007, 411:6-10.

20. Rozin P: "Taste-smell confusions" and the duality of the olfactory sense. Percept Psychophys 1982, 31:397-401.

21. Murphy C, Cain WS, Bartoshuk LM: Mutual action of taste and olfaction. Sens Processes 1977, 1:204-211.

22. Bull T: Taste and the chorda tympani. J Laryngol Otol 1966, 79:479-493.

23. Deems DA, Doty RL, Settle RG, Moore-Gillon V, Shaman P, Mester AF, Kimmelman CP, Brightman VJ, Snow JB Jr: Smell and taste disorders, a study of 750 patients from the University of Pennsylvania Smell and Taste Center. Arch Otolaryngol Head Neck Surg 1991, 117:519-528.

24. Gent JF, Goodspeed RB, Zagraniski RT, Catalanotto FA: Taste and smell problems: validation of questions for the clinical history. Yale $J$ Biol Med 1987, 60:27-35.

25. Stevenson RJ, Langdon R: Olfactory and gustatory hallucinations. In Hallucinations: Research and Practice. Edited by: Blom JD, Sommer IEC. New York: Springer;

26. Kubovy M, Van Valkenburg D: Auditory and visual objects. Cognition 2001, 80:97-126.

27. Bingham AF, Birch GG, de Graaf C, Behan JM, Perring KD: Sensory studies with sucrose-maltol mixtures. Chem Sens 1990, 15:447-456.

28. Frasnelli J, Heilmann S, Hummel T: Responsiveness of human nasal mucosa to trigeminal stimuli depends on the site of stimulation. Neurosci Lett 2004, 362:65-69.

29. Laska M, Distel H, Hudson R: Trigeminal perception of odourant quality in congenital anosmic subjects. Chem Sens 1997, 22:447-456.

30. Scott JW, Acevedo HP, Sherrill L, Phan M: Responses of the rat olfactory epithelium to retronasal air flow. J Neurophysiol 2007, 97:1941-1950.

31. Heilmann S, Hummel T: A new method for comparing orthonasal and retronasal olfaction. Behav Neurosci 2004, 118:412-419.

32. Small DM, Gerber JC, Mak YE, Hummel T: Differential neural responses evoked by orthonasal versus retronasal odorant perception in humans. Neuron 2005, 47:593-605.

33. Mainland J, Sobel N: The sniff is part of the olfactory percept. Chem Sens 2006, 31:181-196.

34. Green BG: Studying taste as a cutaneous sense. Food Qual Prefer 2002, 14:99-109.

35. Todrank J, Bartoshuk LM: A taste illusion: taste sensation localized by touch. Physiol Behav 1991, 50:1027-1031.

36. Lim J, Johnson MB: Potential mechanisms of retronasal odor referral to the mouth. Chem Sens 2011, 36:283-289.
37. Stevenson RJ, Oaten MJ, Mahmut MK: The role of taste and oral somatosensation in olfactory localisation. Q J Exp Psychol 2010, 64:224-240.

38. Stevenson RJ, Mahmut MK, Oaten MJ: The role of attention in the localization of odors to the mouth. Atten Percept Psychophys 2011, 73:247-258.

39. Stevenson RJ, Mahmut MK: Olfactory test performance and its relationship with the perceived location of odors. Atten Percept Psychophys 2011, 73:1966-1976.

40. Spence C, McGlone FP, Kettenmann B, Kobal G: Attention to olfaction: a psychophysical investigation. Exp Brain Res 2001, 138:432-437.

41. Tham WP, Stevenson RJ, Miller LA: The functional role of the mediodorsal thalamic nucleus in human olfaction. Brain Res Rev 2009, 62:109-126.

42. Tham WP, Stevenson RJ, Miller LA: The role of the mediodorsal thalamic nucleus in human olfaction. Neurocase 2011, 17:148-159.

43. Tham WW, Stevenson RJ, Miller LA: The impact of mediodorsal thalamic lesions on olfactory attention and flavor perception. Brain Cogn 2011, 77:71-79.

44. Stevenson RJ, Boakes RA, Wilson JP: Resistance to extinction of conditioned odor perceptions: evaluative conditioning is not unique. J Exp Psychol Learn Mem Cognit 2000, 26:423-440.

45. Issanchou S, Valentin D, Sulmont C, Degel J, Koster EP: Testing odor memory: incidental versus intentional learning, implicit versus explicit memory. In Olfaction, Taste and Cognition. Edited by: Rouby C, Schaal B, Dubois D, Gervais R, Holley A. Cambridge: Cambridge University Press; 2002:211-230.

46. Degel J, Koster EP: Odors: Implicit memory and performance effects. Chem Sens 1999, 24:317-325.

47. Spence C, Nicholls ME, Driver J: The cost of expecting events in the wrong sensory modality. Percept Psychophys 2001, 63:330-336.

48. Prescott J, Murphy S: Inhibition of evaluative and perceptual odor-taste learning by attention to the stimulus elements. Q J Exp Psychol 2009, 62:2133-2140.

49. Stevenson RJ, Mahmut MK: Discriminating the stimulus elements during human odor-taste learning: a successful analytic stance does not eliminate learning. J Exp Psychol Anim Behav Process .

50. Corbetta M, Shulman GL: Control of goal directed and stimulus driven attention in the brain. Nat Rev Neurosci 2002, 3:201-215.

51. Posner Ml: Orienting of attention. The VIlth Sir Frederic Bartlett lecture. Q J Exp Psychol 1980, 32A:3-25.

52. Veldhuizen MG, Shepard TG, Wang MF, Marks LE: Coactivation of gustatory and olfactory signals in flavor perception. Chem Sens 2010, 35:121-133.

doi:10.1186/2044-7248-1-2

Cite this article as: Stevenson: The role of attention in flavour perception. Flavour 2012 1:2

\section{Submit your next manuscript to BioMed Central and take full advantage of:}

- Convenient online submission

- Thorough peer review

- No space constraints or color figure charges

- Immediate publication on acceptance

- Inclusion in PubMed, CAS, Scopus and Google Scholar

- Research which is freely available for redistribution

Submit your manuscript at www.biomedcentral.com/submit
C Biomed Central 